\title{
Radar Waveform Design for Extended Target Recognition under Detection Constraints
}

\author{
Huadong Meng, Yimin Wei, Xuhua Gong, \\ Yimin Liu, and Xiqin Wang \\ Department of Electronic Engineering, Tsinghua University, Beijing 100084, China \\ Correspondence should be addressed to Yimin Wei, weiyimin@gmail.com
}

Received 7 July 2011; Revised 19 October 2011; Accepted 2 November 2011

Academic Editor: J. Rodellar

Copyright (c) 2012 Huadong Meng et al. This is an open access article distributed under the Creative Commons Attribution License, which permits unrestricted use, distribution, and reproduction in any medium, provided the original work is properly cited.

We address the problem of radar phase-coded waveform design for extended target recognition in the presence of colored Gaussian disturbance. Phase-coded waveforms are selected since they can fully exploit the transmit power with sufficient variability. An important constraint, target detection performance, is considered to meet the practical requirements. The waveform is designed to achieve maximum recognition performance under a control on the achievable signal-to-noise ratio (SNR) of every possible target hypothesis. We formulate the code design in terms of a nonconvex, NP-hard quadratic optimization problem in the cases of both continuous and discrete phases. Techniques based on semidefinite relaxation (SDR) and randomization are proposed to approximate the optimal solutions. Simulation results show that the recognition performance and the detection requirements are well balanced and accurate approximations are achieved.

\section{Introduction}

Cognitive radar is a newly proposed radar system concept, in which waveform-agile sensing can be realized with the featured feedback structure [1]. Based on the prior knowledge about targets and environments, transmit signals can be adaptively optimized to improve system performance and efficiency. Inspired by this concept, many attempts have been focusing on target recognition using waveform adaptation. In [2], Goodman et al. proposed the integration of waveform design techniques with a sequential hypothesis testing (SHT) framework [3] that controls when hard decisions may be made with adequate confidence [4]. They also compared two different waveform design techniques for use with active sensors operating in a target recognition application. One is considered by Bell [5] based on a maximization of the mutual information between a random target ensemble and the echo signal, while the other is based on maximizing the weighted average Euclidean 
distance or Mahalanobis distance (in additive colored noise) between the ideal echoes from different target hypotheses [6,7], where known impulse responses are used to model the target scattering behaviors. In [8], the optimum Multiinput Multioutput (MIMO) target identification problem was formulated as a quadratic optimization, which derived a solution with unconstrained amplitude based on the eigenvalue decomposition method.

However, some practical constraints must be considered in the recognition waveform design. The first is constant waveform modulus. From the standpoint of hardware realization [9], modulus constraint is more suitable than the total energy constraint considered in [2]. Waveforms were restricted to be constant modulus in recent researches, including singlefrequency signals [10] and phase-coded signals [11,12], to fully exploit the transmit power in the pulse duration. Meanwhile, the use of nonlinear frequency modulated (NLFM) waveform to achieve the constant modulus based on the stationary phase method was also discussed in [8], where it is difficult to obtain the designed optimal signal in accordance with the arbitrary energy spectral density (ESD) or autocorrelation function. The second constraint is the detection performance. Detection is absolutely an essential prerequisite for any estimation or recognition task in a radar system [13]. The signal model in [2] can only be applied to the situations with high signal-to-noise ratios (SNRs), in which target detection may not be a problem. In the situations that detection performances are critical, target output SNRs must be ensured in the waveform design to meet the detection requirements.

In this paper, we focus on the phase-coded waveform design for extended target recognition in the presence of colored Gaussian disturbance. The phase code is optimized according to the following criterion: maximization of the recognition performance under a control on the output SNR of every possible target hypothesis. Because of the stringent constraint on each target hypothesis, or with the increase in the number of target hypotheses, the detection requirements may not be met simultaneously for all the possible targets. To measure the impact of the detection constraints on the optimization, a preanalysis is performed, in which the maximum achievable SNR for all the hypotheses is acquired. The question of whether our detection-constrained optimization problem has a nonempty feasible region is clearly determined by the maximum achievable SNR and our desired SNR. If the desired SNR is smaller than the maximum achievable SNR, optimal solution exists. Otherwise, no solution can be found. Taking into account the modulus constraint and the detection SNR threshold, we formulate the code design in terms of a nonconvex, NP-hard quadratic optimization problem in the cases of both continuous and discrete phases. A novel and computationally attractive method, which is referred to as Semidefinite Relaxation (SDR) and randomization [14, 15], is presented to approximate the optimal solutions. The SDR technique can be applied to many nonconvex quadratically constrained quadratic programs (QCQPs) in an almost mechanical fashion [16]. Many practical experiences have indicated that SDR is capable of providing accurate approximations to the QCQPs $[17,18]$. The SDR method first relaxes the NP-hard quadratic optimization problem to a convex optimization problem by abandoning the rank one constraint on the waveform autocorrelation matrix. After the relaxation, the relaxed problem can be solved by using the semidefinite programming (SDP) with polynomial complexity [19]. The obtained autocorrelation matrix is then used to randomly generate the feasible solutions for the original problem. The solution with maximum weighted average distance is selected to be the result. Taking the advantage of the randomization, the proposed approach can avoid being trapped in local optima. Compared with the other methods $[8,11,12]$, the accurate approximations can be achieved with a modest number of randomizations. In addition, since SDR expands the feasible region of the original problem and keeps any other condition unchanged, the result of the relaxed 
problem can be used as an upper bound for the optimization. Simulation results show that the performance of the approximate solution is very close to the upper bound.

\section{System Model}

We consider the target recognition problem in which one of $M$ possible targets may appear [2]. Our objective is to identify the target under a control on the detection SNR of every target hypothesis. The transmit phase-coded waveform is denoted by

$$
\mathbf{s}=[s(1), s(2), \ldots, s(N)]^{T}=\left[\exp \left(j \varphi_{1}\right), \exp \left(j \varphi_{2}\right), \ldots, \exp \left(j \varphi_{N}\right)\right]^{T},
$$

where $(\cdot)^{T}$ is the transpose operator, $\varphi_{n}$ denotes the phase of the $n$th entry $s(n)$, and $N$ is the length of the code. Each target hypothesis $H_{i}, i \in\{1,2, \ldots, M\}$ has a fixed impulse response $\mathbf{h}_{i}=\left[h_{i}(1), h_{i}(2), \ldots, h_{i}(L)\right]^{T}$ which is exactly known. If the $i$ th target is present, the corresponding echo signal is given by

$$
\mathbf{y}=\mathbf{h}_{i} * \mathbf{s}+\mathbf{n},
$$

where the disturbance vector $\mathbf{n}$ is a zero-mean complex circular Gaussian vector with known positive definite covariance matrix $\mathbf{R}$ and $*$ denotes the convolution operator. The convolution operation in (2.2) can be replaced with matrix multiplication by defining the convolution matrix

$$
\mathbf{Q}_{i}=\left[\begin{array}{cccc}
h_{i}(1) & 0 & \cdots & 0 \\
h_{i}(2) & h_{i}(1) & \ddots & \vdots \\
\vdots & h_{i}(2) & \ddots & \vdots \\
h_{i}(L) & \vdots & \ddots & 0 \\
0 & h_{i}(L) & \ddots & h_{i}(1) \\
\vdots & 0 & \ddots & h_{i}(2) \\
\vdots & \vdots & \ddots & \vdots \\
0 & 0 & 0 & h_{i}(L)
\end{array}\right]
$$

which is an $L+N-1$ by $N$ complex matrix. Equation (2.2) can therefore be written as

$$
\mathbf{y}=\mathbf{Q}_{i} \mathbf{s}+\mathbf{n} .
$$

\section{Phase Code Design Criteria}

A waveform design technique was proposed by Goodman et al. [2] to optimize the transmit waveform under the energy constraint $\mathbf{s}^{H} \mathbf{s} \leq E$, where $\mathbf{s}$ is considered as an arbitrary waveform, $(\cdot)^{H}$ is the Hermitian transpose operator, and $E$ is the transmit energy. He extended the provably optimal two-target-hypothesis recognition waveform [6] to 
a multihypothesis situation. The idea of the algorithm is to maximize the weighted average Euclidean distance or Mahalanobis distance (in additive colored noise) between the ideal echoes from different hypotheses, where the weighting coefficients are designed according to the prior probabilities of target hypotheses. The mathematical representation of the algorithm is given by

$$
\begin{aligned}
\max & \mathbf{s}^{H} \boldsymbol{\Omega} \mathbf{s} \\
\text { s.t. } & \mathbf{s}^{H} \mathbf{s} \leq E,
\end{aligned}
$$

where the weighted target correlation matrix $\Omega$ is defined as

$$
\boldsymbol{\Omega}=\sum_{i=1}^{M-1} \sum_{j=i+1}^{M} P_{i} P_{j}\left(\mathbf{Q}_{i}-\mathbf{Q}_{j}\right)^{H} \mathbf{R}^{-1}\left(\mathbf{Q}_{i}-\mathbf{Q}_{j}\right)
$$

where $P_{i}$ and $P_{j}$ are the prior probabilities of hypothesis $H_{i}$ and $H_{j}$. Under the total energy constraint, the maximization problem can be easily solved. The optimal solution of (3.1) is proportional to the eigenvector corresponding to the largest eigenvalue of $\boldsymbol{\Omega}$. Unfortunately, the eigenvector does not usually have constant modulus and cannot guarantee the output SNR for all the possible targets.

To meet the practical requirements, we modify the model by considering two additional constraints, constant waveform modulus and target detection performance. As shown in (2.1), we restrict the transmit waveform to a phase code that has constant waveform modulus. Phase codes can fully exploit the transmit power in the pulse duration with sufficient variability, which makes the optimization possible.

The target detection problem has been previously studied by Bell in [5]. He pointed out that the optimal receiver filter that maximizes the output SNR for a given transmit waveform $\mathbf{s}$ is equal to $\mathbf{R}^{-1} \mathbf{Q s}$, and the corresponding SNR is given by $\mathbf{s}^{H} \mathbf{Q}^{H} \mathbf{R}^{-1} \mathbf{Q s}$, where $\mathbf{Q}$ is the convolution matrix of a specific extended target. Since the optimal receiver filter is related to target property $\mathbf{Q}$, a unified receiver filter, for example, the traditional matched filter $\mathbf{R}^{-1} \mathbf{s}$, is no longer appropriate for the multihypothesis extended target situation. For the radars that aim to detect and identify several target hypotheses, an intuitive detection method is to use all the possible receiver filters $\mathbf{R}^{-1} \mathbf{Q}_{i} \mathbf{s}$ in the detection procedure. If the convolution of the echo signal with any of the receiver filter exceeds the detection threshold, a positive decision is made. Under this strategy, to ensure the output SNR of every possible target hypothesis, the transmit waveform should be designed to satisfy

$$
\mathbf{s}^{H} \mathbf{O}_{i} \mathbf{s} \geq d, \quad i \in\{1,2, \ldots, M\},
$$

where $\mathbf{O}_{i}=\mathbf{Q}_{i}^{H} \mathbf{R}^{-1} \mathbf{Q}_{i}$ and $d$ is the desired SNR. The criteria in (3.3) are universal to different detection methods, since it controls the lower limit of the achievable SNR at the receiver with no limitation on the specific algorithm. It is obviously a necessary condition for any detection strategy. We also consider two possibilities for the entries of $\mathbf{s}$. One is the continuous phase code that $\varphi_{n}$ can take any value between 0 and $2 \pi$, namely, the modulus of the $n$th entry $|s(n)|=1, n=1, \ldots, N$. The other is a discrete phase code with quantization interval $2 \pi / D$, which can be expressed as $s(n) \in\left\{1, e^{j 2 \pi / D}, \ldots, e^{j 2 \pi(D-1) / D}\right\}, n=1, \ldots N$. 


\section{Phase Coding Algorithm under Detection Constraints}

In this section, we formulate the continuous phase-coded waveform design in terms of the following complex quadratic optimization problem:

$$
\begin{aligned}
\max & \mathbf{s}^{H} \mathbf{\Omega} \mathbf{s} \\
\text { s.t. } & \mathbf{s}^{H} \mathbf{O}_{i} \mathbf{s} \geq d, \quad i \in\{1,2, \ldots, M\}, \\
& |s(n)|=1, \quad n=1, \ldots, N .
\end{aligned}
$$

The objective function in this problem is the same as the one in [2], which aims to maximize the weighted average Mahalanobis distance between the ideal echoes from different hypotheses. In contrast with the problem in [2], additional practical constraints are considered. The modulus of the waveform is restricted to be a constant, which equals 1 in this case. The detection constraints require that the achievable SNR for each target hypothesis be larger than the desired SNR $d$. The feasible region in (4.1) highly depends on the target properties and the noise characteristic. In the case of weak disturbance, the demands for detection are easily met. Feasible region is slightly affected by the detection constraints. The problem may reduce to a simpler quadratic optimization problem shown as

$$
\begin{aligned}
\max & \mathbf{s}^{H} \mathbf{\Omega s} \\
\text { s.t. } & |s(n)|=1, \quad n=1, \ldots, N,
\end{aligned}
$$

which is quite similar to the case studied in [2], except the constant modulus constraint. In the case that detection requirements are critical, the constraints in (4.1) are necessary and important. The solution of the problem is highly dependent on these constraints. In some extreme cases, the feasible region may appear to be an empty set because of the stringent constraints. It means that no matter what phase code is used, the desired SNRs cannot be achieved simultaneously for all the target hypotheses. To meet most of the goals, one may need to relax the constraints on the hypotheses with low prior probabilities. It is necessary to perform a preanalysis on the detection constraints. By solving the following optimization problem:

$$
\begin{aligned}
\max & t \\
\text { s.t. } & \mathbf{s}^{H} \mathbf{O}_{i} \mathbf{s} \geq t, \quad i \in\{1,2, \ldots, M\}, \\
& |s(n)|=1, \quad n=1, \ldots N,
\end{aligned}
$$

one can acquire the maximum achievable SNR for all the target hypotheses, which is a boundary point for the desired SNR $d$. If $d$ is larger than $t$, the feasible region in (4.1) is empty. Concessions must be made in the constraints, or no solution can be obtained. If $d$ is much smaller than $t$, the resulting value of (4.1) may be close to that of (4.2). We use the ratio between $d$ and $t$ to measure the impact of the detection constraints on the resulting value. For those situations that approximately satisfy $0.1<d / t<1$, the optimization problem in (4.1) is very important.

Since both the problems in (4.1) and (4.3) are nonconvex and NP-hard, one cannot find polynomial time algorithms for computing the optimal solutions. As a consequence, our 
goal is to find an efficient algorithm for approximating the solutions. Semidefinite Relaxation (SDR) and randomization technique can be applied to such nonconvex quadratically constrained quadratic programs, and it is capable of providing accurate approximations [16]. By defining the matrix $\mathbf{Z}=\mathbf{s} \mathbf{s}^{H}$, the problem in (4.1) can be written in the following form:

$$
\begin{array}{ll}
\max & \operatorname{tr}(\boldsymbol{\Omega Z}) \\
\text { s.t. } & \operatorname{tr}\left(\mathbf{O}_{i} \mathbf{Z}\right) \geq d, \quad i \in\{1,2, \ldots, M\}, \\
& Z(n, n)=1, \quad n=1, \ldots, N, \\
& \mathbf{Z} \geq 0, \quad \operatorname{rank}(\mathbf{Z})=1,
\end{array}
$$

where $\operatorname{tr}(\cdot)$ denotes the trace of a matrix, $Z(n, n)$ denotes the $(n, n)$ th entry of $\mathbf{Z}, \mathbf{Z} \geq 0$ indicates that $\mathbf{Z}$ is positive semidefinite, and $\operatorname{rank}(\cdot)$ denotes the rank of a matrix. The objective function and all the constraints in (4.4) are convex in $\mathbf{Z}$, except the rank constraint $\operatorname{rank}(\mathbf{Z})=1$. By abandoning the rank constraint, the problem can be relaxed to a convex optimization problem as follows:

$$
\begin{array}{ll}
\max & \operatorname{tr}(\boldsymbol{\Omega Z}) \\
\text { s.t. } & \operatorname{tr}\left(\mathbf{O}_{i} \mathbf{Z}\right) \geq d, \quad i \in\{1,2, \ldots, M\}, \\
& Z(n, n)=1, \quad n=1, \ldots, N, \\
& \mathbf{Z} \geq 0 .
\end{array}
$$

It can be solved by using the semidefinite programming (SDP) to any arbitrary accuracy with polynomial complexity in the problem size $N$ and the number of constraints $M$. The complexity also depends on the required solution accuracy.

The next issue that must be addressed is how to convert a global optimal solution $\widehat{\mathbf{Z}}$ to (4.5) into a feasible solution $\mathbf{s}$ to (4.1) [16]. If the rank of $\widehat{\mathbf{Z}}$ equals one, the $\mathbf{s}$ that satisfies $\widehat{\mathbf{Z}}=\mathbf{s} \mathbf{s}^{H}$ is the global optimal solution. It means that the relaxation in the feasible region does not change the maximum point of (4.4). In general, the rank of $\widehat{Z}$ is greater than one. Feasible s must be extracted from $\widehat{\mathbf{Z}}$. Intuitively, a vector $\gamma$ randomly generated from a zeromean complex normal distribution with covariance matrix $\widehat{\mathbf{Z}}$ can be used as an approximate solution. To meet the constant modulus constraint, we map $r$ to a constant modulus vector via $\mathbf{s}=\exp [j \arg (r)]$, where the function $\arg (\cdot)$ returns the phase angles of a complex vector in $[0,2 \pi)$. If $\mathbf{s}$ meets all the detection constraints in (4.1), it is an eligible approximation. Otherwise, another randomization step is required. In order to improve the approximation quality, the randomization step is repeated several times. The eligible s yielding the largest objective function value is chosen as the approximate solution.

Since the relaxation procedure in (4.5) expands the feasible region of the original problem and keeps any other condition unchanged, the acquired objective function $\operatorname{tr}(\boldsymbol{\Omega} \widehat{\mathbf{Z}})$ can be used as an upper bound for (4.1). Simulation results show that with a modest number of randomizations, the approximate solution is very close to the upper bound. In other words, accurate approximation is achieved. 
Problem (4.3) also can be solved by using the same relaxation and randomization method. It is relaxed to the following convex optimization form:

$$
\begin{array}{ll}
\max & t \\
\text { s.t. } & \operatorname{tr}\left(\mathbf{O}_{i} \mathbf{Z}\right) \geq t, \quad i \in\{1,2, \ldots, M\}, \\
& \mathrm{Z}(n, n)=1, \quad n=1, \ldots, N, \\
& \mathrm{Z} \geq 0 .
\end{array}
$$

Same mapping method is used to randomly obtain the constant modulus vector $\mathbf{s}$ from the global optimal solution $\widehat{\mathbf{Z}}_{t}$ to (4.6). The $\mathbf{s}$ that maximizes the minimum achievable SNR for all the target hypotheses is selected to be the approximate solution, and the corresponding objective function $t$ is acquired to be the boundary point for the desired SNR $d$.

The complete algorithm to approximate the optimal phase code is summarized as follows. It consists of two parts, estimating the maximum achievable SNR $t$ and finding the optimal phase code $\mathbf{s}$.

Algorithm 4.1 (Estimating the maximum achievable SNR). (1) Solve the SDP problem below and denote by $\widehat{\mathbf{Z}}_{t}$ an optimal solution

$$
\begin{array}{ll}
\max & t \\
\text { s.t. } & \operatorname{tr}\left(\mathbf{O}_{i} \mathbf{Z}\right) \geq t, \quad i \in\{1,2, \ldots, M\} \\
& Z(n, n)=1, \quad n=1, \ldots, N \\
& \mathbf{Z} \geq 0 .
\end{array}
$$

(2) Generate random vectors $\gamma_{k}, k \in\{1,2, \ldots, K\}$ from the complex normal distribution $\mathbb{N}_{\mathbb{C}}\left(\mathbf{0}, \widehat{\mathbf{Z}}_{t}\right)$, where $K$ is the number of randomizations.

(3) Assign each $\mathbf{s}_{k}=\exp \left[j \arg \left(r_{k}\right)\right]$. The maximum achievable SNR is then given by

$$
t=\max _{k \in\{1,2, \ldots, K\}}\left[\min _{i \in\{1,2, \ldots, M\}}\left(\mathbf{s}_{k}^{H} \mathbf{O}_{i} \mathbf{s}_{k}\right)\right]
$$

Algorithm 4.2 (Finding the optimal phase code). (1) If the required SNR $d>t$, the feasible region is empty. Concessions must be made in theconstraints, or no solution can be acquired.

(2) Solve the SDP problem below and denote by $\widehat{\mathbf{Z}}$ an optimal solution

$$
\begin{array}{ll}
\max & \operatorname{tr}(\boldsymbol{\Omega Z}) \\
\text { s.t. } & \operatorname{tr}\left(\mathbf{O}_{i} \mathbf{Z}\right) \geq d, \quad i \in\{1,2, \ldots, M\} \\
& Z(n, n)=1, \quad n=1, \ldots N \\
& \mathbf{Z} \geq 0 .
\end{array}
$$


(3) Generate random vectors $\gamma_{k}, k \in\{1,2, \ldots, K\}$ from the complex normal distribution $\mathbb{N}_{\mathbb{C}}(\mathbf{0}, \widehat{\mathbf{Z}})$.

(4) Assign each $\mathbf{s}_{k}=\exp \left[j \arg \left(\boldsymbol{\gamma}_{k}\right)\right]$. The feasible $\mathbf{s}_{k}$ that satisfies $\mathbf{s}_{k}^{H} \mathbf{O}_{i} \mathbf{s}_{k} \geq d, i \in$ $\{1,2, \ldots, M\}$ yielding the largest objective function is chosen as the approximate solution, which is shown as

$$
\begin{gathered}
U=\left\{k: \mathbf{s}_{k}^{H} \mathbf{O}_{i} \mathbf{s}_{k} \geq d \forall 1 \leq i \leq M ; 1 \leq k \leq K\right\}, \\
\mathbf{s}=\underset{\mathbf{s}_{k}, k \in U}{\arg \max }\left(\mathbf{s}_{k}^{H} \boldsymbol{\Omega} \mathbf{s}_{k}\right) .
\end{gathered}
$$

\section{Discrete Phase Coding Algorithm under Detection Constraints}

In this section, we focus on a more constrained problem, discrete phase code design, that the phase of the code can only take several values equally spaced in $[0,2 \pi)$. For example, the phase of a binary phase code is selected from $\{0, \pi\}$ and the phase alphabet of a quadrature phase code is $\{0, \pi / 2, \pi, 3 \pi / 2\}$. For an alphabet with quantization interval $2 \pi / D$, the phasecoded waveform design is formulated as follows:

$$
\begin{array}{ll}
\max & \mathbf{s}^{H} \boldsymbol{\Omega} \mathbf{s} \\
\text { s.t. } & \mathbf{s}^{H} \mathbf{O}_{i} \mathbf{s} \geq d, \quad i \in\{1,2, \ldots, M\}, \\
& s(n) \in\left\{1, e^{j 2 \pi / D}, \ldots, e^{j 2 \pi(D-1) / D}\right\}, \quad n=1, \ldots N .
\end{array}
$$

Compared to the continuous phase design in (4.1), the feasible region in (5.1) is even smaller. The objective function of (4.1) can be used as an upper bound for (5.1), since discrete phase code is a subset of continuous phase code. For the same reason, if an alphabet size $D_{1}$ is an integer multiple of another size $D_{2}$, the objective function of (5.1) with size $D_{1}$ is definitely larger than that with $D_{2}$. We can further speculate that the objective function in (5.1) increases monotonically with increasing alphabet size $D$, even in the situations that alphabet sizes are coprime.

The SDR algorithm we presented in the previous section can be applied to (5.1), if some refinements are made in the mapping procedure. We use the following mapping rule to replace the continuous one given by $\mathbf{s}=\exp [j \arg (r)]$ :

$$
\mathbf{s}=\exp \left[j\left(\frac{2 \pi}{D}\right) \cdot\left\lfloor\frac{\arg (\boldsymbol{r})}{(2 \pi / D)} \mid\right]\right.
$$

The idea above is simple and clear. Continuous phases $\arg (\gamma)$ are rounded down to the nearest discrete phase values in $\{0,2 \pi / D, \ldots, 2 \pi(D-1) / D\}$ to adapt to the requirements of discrete phases. One may notice that the mapping is not "unbiased." A round-down is applied rather than the "unbiased" round to nearest. In fact, these methods have exactly the same performance, even for round-up. It is because the bias here can be treated as an initial phase of the waveform, and the initial phase does not affect the result. 
The complete algorithm is summarized in Algorithm 5.1.

Algorithm 5.1 (Finding the optimal discrete phase code). (1) Solve the SDP problem below and denote by $\widehat{\mathbf{Z}}$ an optimal solution

$$
\begin{aligned}
\max & \operatorname{tr}(\boldsymbol{\Omega Z}) \\
\text { s.t. } & \operatorname{tr}\left(\mathbf{O}_{i} \mathbf{Z}\right) \geq d, \quad i \in\{1,2, \ldots, M\} \\
& Z(n, n)=1, \quad n=1, \ldots, N \\
& \mathbf{Z} \geq 0 .
\end{aligned}
$$

(2) Generate random vectors $r_{k}, k \in\{1,2, \ldots, K\}$ from the complex normal distribution $\mathbb{N}_{\mathbb{C}}(\mathbf{0}, \widehat{\mathbf{Z}})$.

(3) Assign each $\mathbf{s}_{k}=\exp \left[j(2 \pi / D) \cdot\left[\arg \left(r_{k}\right) /(2 \pi / D)\right]\right]$. The feasible $\mathbf{s}_{k}$ that satisfies $\mathbf{s}_{k}^{H} \mathbf{O}_{i} \mathbf{s}_{k} \geq d, i \in\{1,2, \ldots, M\}$ yielding the largest objective function is chosen as the approximate solution, which is shown as

$$
\begin{gathered}
U=\left\{k: \mathbf{s}_{k}^{H} \mathbf{O}_{i} \mathbf{s}_{k} \geq d \forall 1 \leq i \leq M, 1 \leq k \leq K\right\}, \\
\mathbf{s}=\underset{\mathbf{s}_{k}, k \in U}{\arg \max }\left(\mathbf{s}_{k}^{H} \boldsymbol{\Omega} \mathbf{s}_{k}\right)
\end{gathered}
$$

In addition, the study on the maximum achievable SNR also can be imported to discrete cases by using the same mapping rule. It will not be repeatedly stated here.

\section{Simulation Results}

In this section, we present simulation results that demonstrate the benefits of the presented algorithms and illustrate the potential consequences of ignoring detection constraints in recognition waveform design. A target set with $M=4$ impulse responses is randomly generated from a flat power spectral density (PSD). The length of the impulse response $L$ equals 12. Once the impulse responses are generated, it is assumed that they are known exactly. The length of the phase code $N$ equals 32 and the covariance matrix $\mathbf{R}=\mathbf{I}$. The initial prior probability $P_{i}$ is set to $1 / M$ for every target hypothesis.

For Figure 1, we first calculate the maximum achievable SNR $t$ for the target set. The required SNR $d$ is then set to $0.8 t$ to simulate the situation that detection requirements are critical. The continuous phase code is designed by using the proposed algorithm with $K=50$ randomizations. The spectra of both the simple recognition waveform (the approximate solution of (4.2)) and the waveform that considers the detection performance (the approximate solution of (4.1)) are compared with the weighted target spectral difference. The weighted target spectral difference is just a representation of the weighted target correlation matrix (3.2) in the frequency domain. Both the weighted target spectral difference and the spectrum of the simple recognition waveform are normalized to their peak power. The spectrum of the detection-constrained waveform is normalized to the simple recognition waveform's peak power to ensure that both the waveforms have the same adjustment scale. As shown in Figure 1, to maximize the weighted Euclidean distance (white noise in this case), the simple recognition waveform focuses most of its energy on the maximum response 


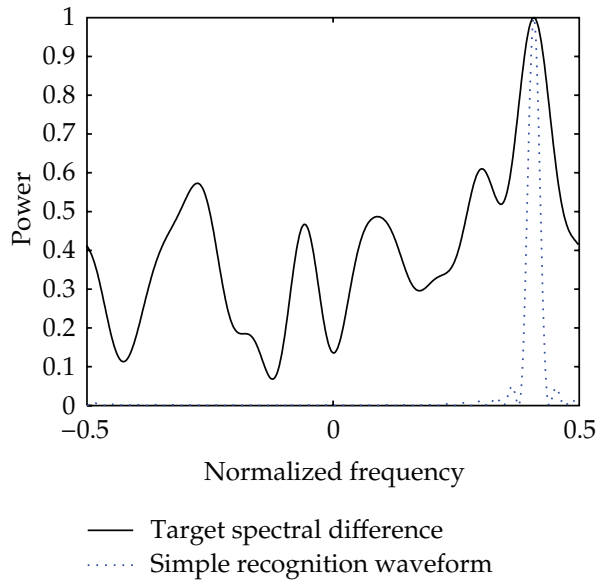

(a)

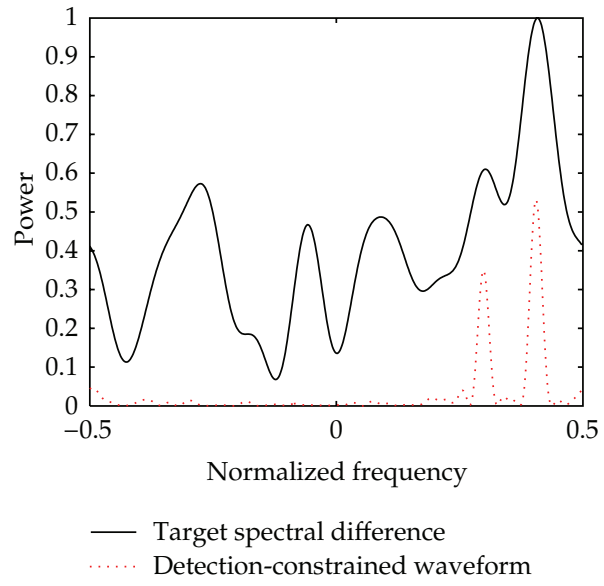

(b)

Figure 1: Waveform spectra (dotted line) compared to target spectral difference (solid line). The spectrum of the simple recognition waveform is shown in (a), and the spectrum of the detection-constrained waveform is shown in (b). The desired SNR $d=0.8 t$.

frequency, since the Fourier transform preserves the Euclidean distance between signal and its spectrum. However, the phase code that considers the detection performance spreads its energy into several narrow bands.

An intuitive explanation of the energy spreading is shown in Figure 2, where the waveform spectra are compared with the spectra of all the four target hypotheses we attempt to discriminate. Both the waveform spectra are normalized to the simple recognition waveform's peak power. The target spectra are normalized to the maximum peak power of the four targets. As we can see in Figure 2(a), since both target no. 1 and no. 4 show weak responses near the energy peak of the simple recognition waveform (normalized frequency 0.4 ), the detection requirements cannot be met for the two. The proposed method distributes some of the energy to the normalized frequency 0.3 (as shown in Figure 2(b)), where the responses of target no. 1 and no. 4 are relatively strong, to enhance the detection SNR. The normalized frequency 0.3 is also the second highest peak of the weighted target spectral difference (as shown in Figure 1(b)). Therefore, the detection requirements are met without sacrificing too much recognition performance in the energy redistribution.

In Figure 3, the desired SNR $d$ is adjusted from $0.5 t$ to $0.8 t$ to show the impact of the detection constraints on the waveform spectrum. Same target set is employed as in Figures 1 and 2. The spectra of the detection-constrained waveforms are also normalized to the simple recognition waveform's peak power. As we can see, with the increasing of the desired SNR the presented algorithm transfers more and more energy from the peak of the target spectral difference (normalized frequency 0.4 ) to the place where target no. 1 and no. 4 have relatively strong responses (normalized frequency 0.3 ) to meet the detection constraints.

The detail of how the objective function and the four detection constraints are affected by the increasing of the desired SNR is shown in Table 1. The performances of the simple recognition waveform $(d=0)$ and the detection-constrained waveforms under different thresholds are listed in Table 1 . The SNR of every sample target is normalized to $t$, and the objective functions are normalized to the simple recognition waveform's objective function. For the simple recognition waveform, the SNRs of target no. 2 and no. 3 are more than twice as large as $t$, while the SNRs of target no. 1 and no. 4 are less than half of $t$. The sample 

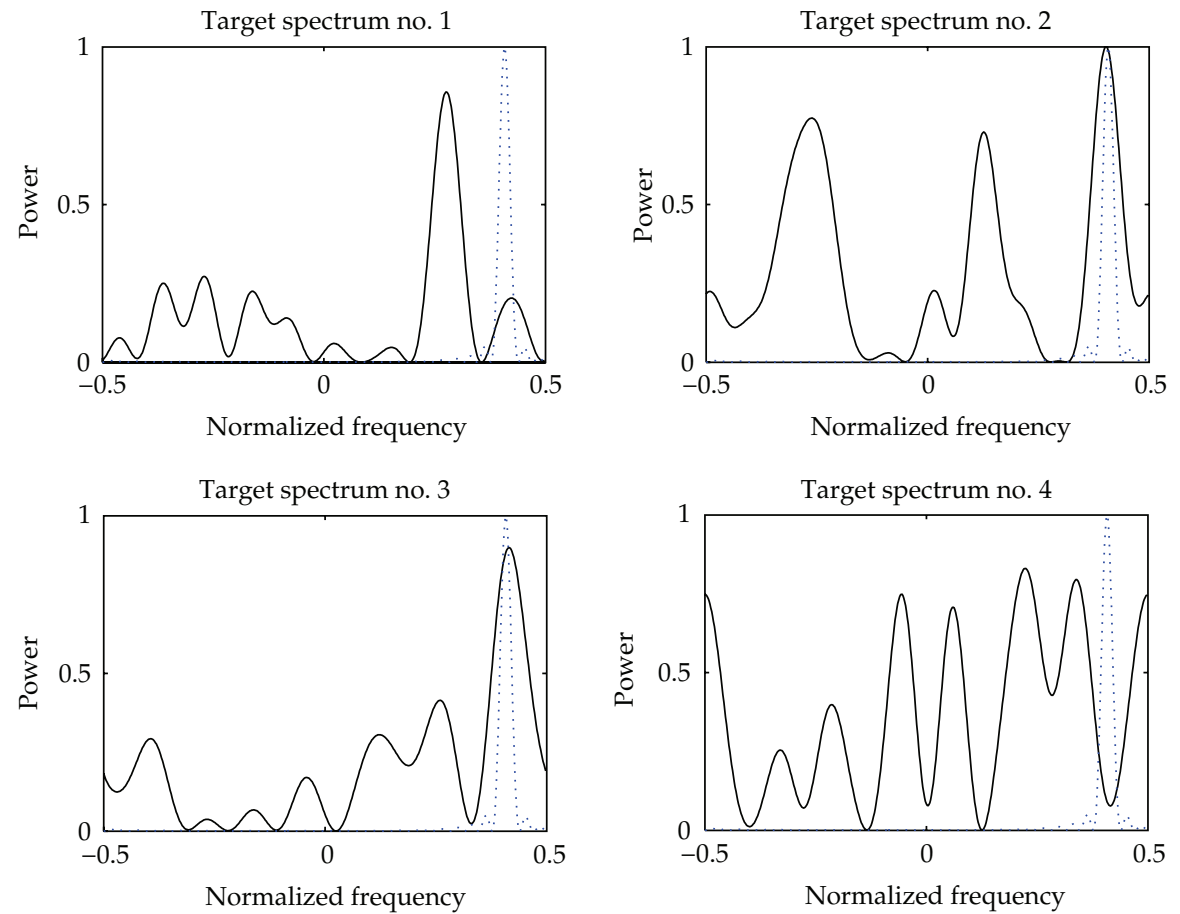

(a)
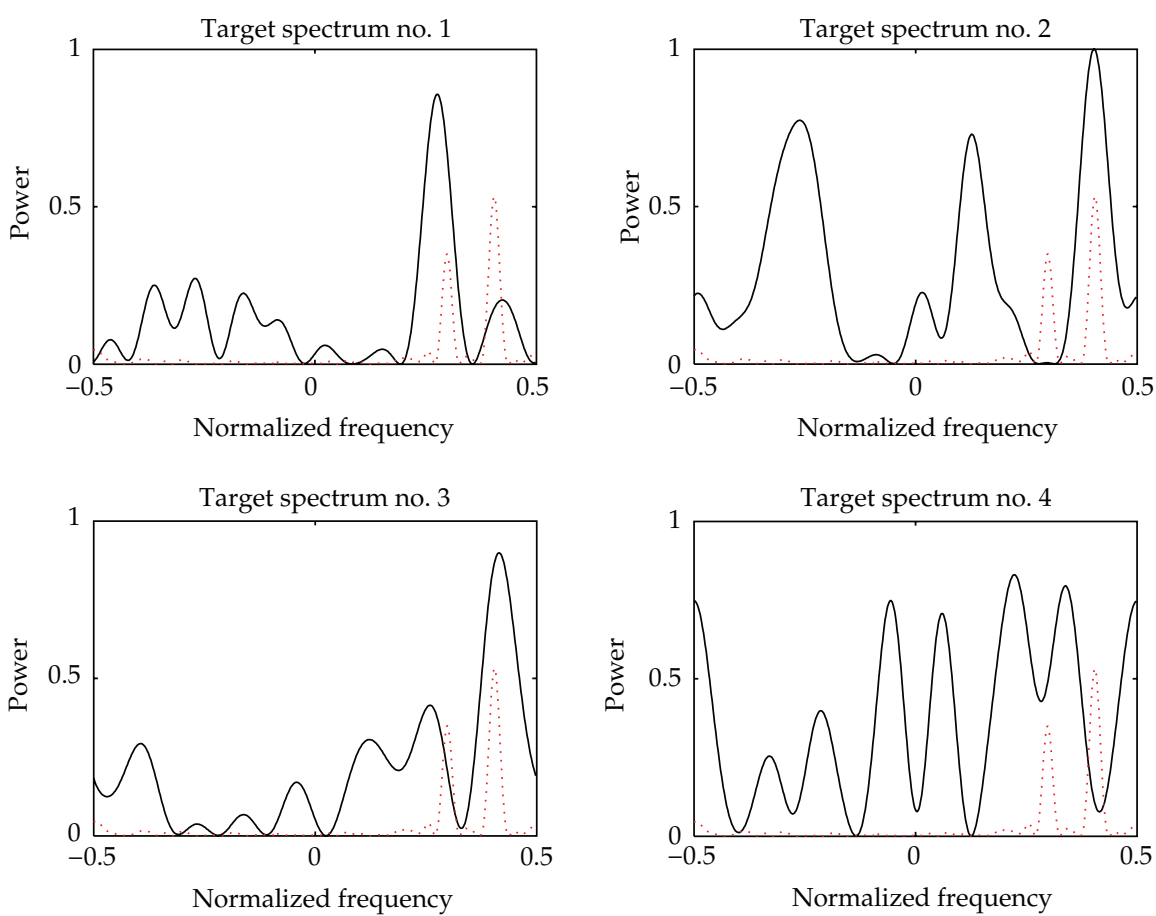

(b)

Figure 2: Waveform spectra (dotted line) compared to sample target spectra (solid line). The spectrum of simple recognition waveform is shown in (a), and the spectrum of the detection-constrained waveform is shown in (b). The desired SNR $d=0.8 t$. 

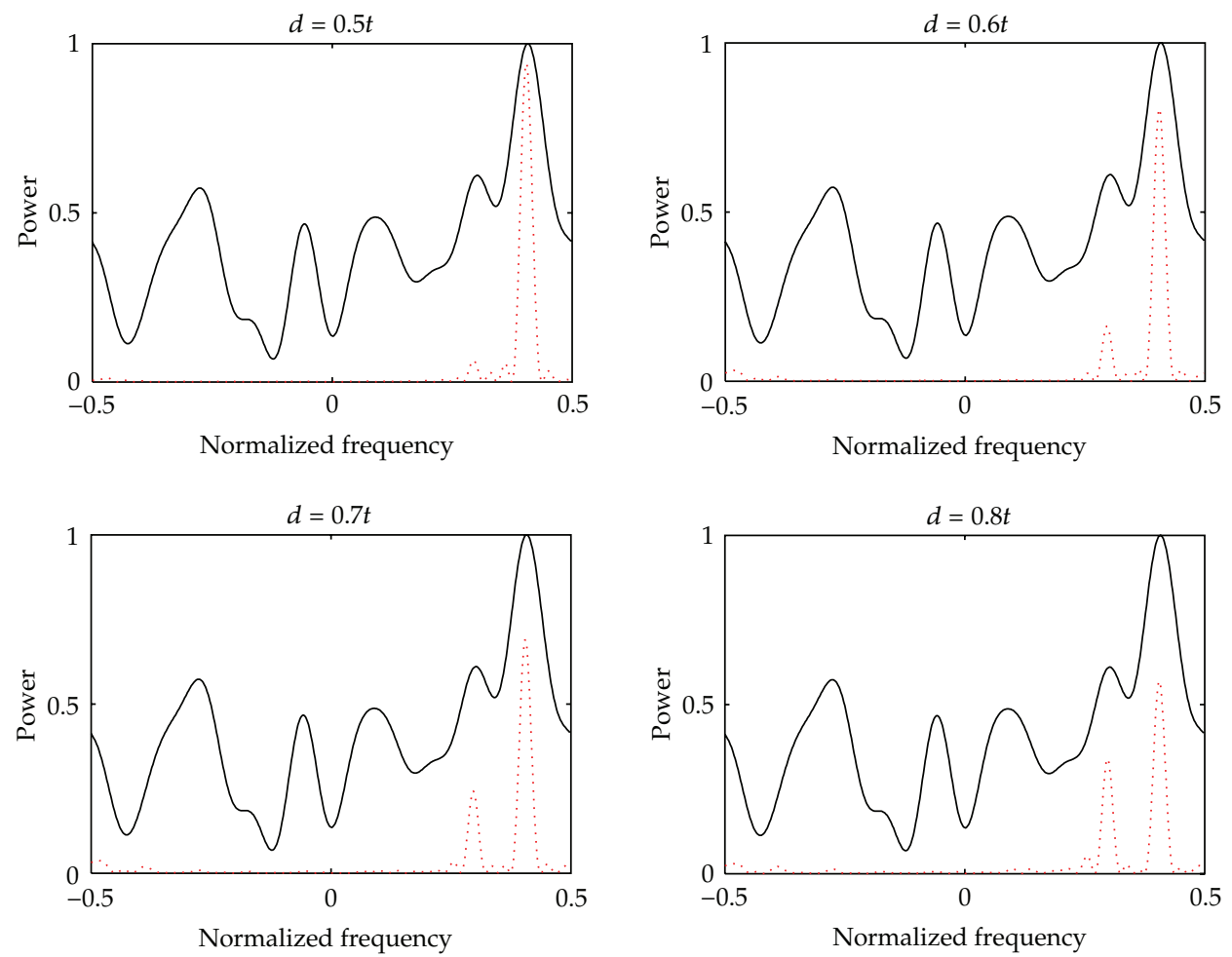

Figure 3: Detection-constrained waveform spectra (dotted line) compared to target spectral difference (solid line) in the case of different desired SNR $d$.

Table 1: objective function and the detection constraints corresponding to the approximate solution under different thresholds.

\begin{tabular}{lccccc}
\hline Threshold & Target no. 1 & Target no. 2 & Target no. 3 & Target no. 4 & Objective function \\
\hline$d=0$ & 0.47 & 2.35 & 2.15 & 0.42 & 1 \\
$d=0.5 t$ & 0.50 & 2.25 & 2.03 & 0.50 & 0.97 \\
$d=0.6 t$ & 0.61 & 1.96 & 1.82 & 0.61 & 0.91 \\
$d=0.7 t$ & 0.70 & 1.71 & 1.64 & 0.71 & 0.85 \\
$d=0.8 t$ & 0.82 & 1.44 & 1.44 & 0.81 & 0.80 \\
\hline
\end{tabular}

targets show significant difference in detection, since only the recognition performance is considered in the optimization. In the case of detection-constrained waveforms, the SNRs of target no. 1 and no. 4 gradually become larger with increasing desired SNR, and always exceed the threshold. Of course, there is no free lunch. Tighter constraints will inevitably lead to a decline in the objective function. Not only the recognition objective function but also the SNRs of target no. 2 and no. 3 decrease with increasing threshold, since part of the transmit energy is used to improve the SNRs of target no. 1 and no. 4. One may also notice that if the desired SNR $d \leq 0.4 t$, the simple recognition waveform is also the optimal solution of the detection-constrained problem, since the detection constraints are already met.

For Figure 4, 500 target sets are randomly generated according to the model used in Figures 1 and 2. For each target set, the continuous phase code design problem is solved 


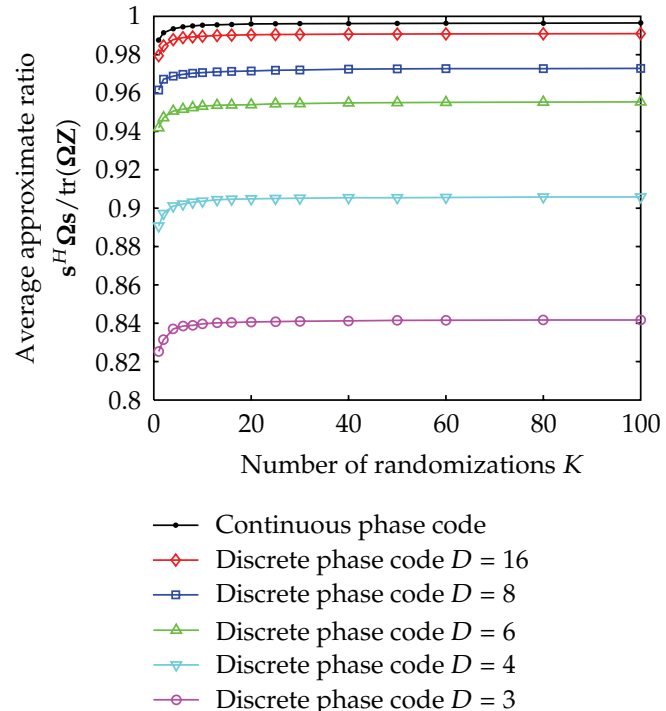

(a)

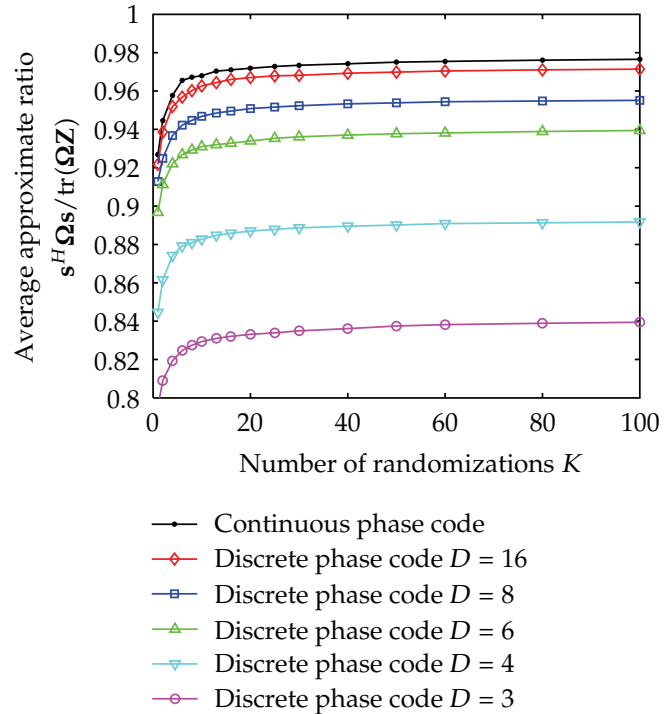

(b)

Figure 4: Average approximate ratio versus number of randomizations. The situation of $d=0.2 t$ is shown in (a), and the situation of $d=0.5 t$ is shown in (b).

and the discrete phase code design problem is solved in different alphabet size $D$. As we mentioned in Section 4, the acquired objective function $\operatorname{tr}(\boldsymbol{\Omega} \widehat{\mathbf{Z}})$ of the relaxed problem (4.5) is an upper bound of the continuous phase code design problem (4.1). Although the optimal solution of (4.1) we attempt to approximate is unknown, the optimal value $v_{\text {opt }}$ is squeezed to $\mathbf{s}^{H} \boldsymbol{\Omega} \mathbf{s} \leq v_{\text {opt }} \leq \operatorname{tr}(\boldsymbol{\Omega} \widehat{\mathbf{Z}})$, where $\mathbf{s}$ is the designed waveform. The ratio between the achieved objective function and the upper bound $r=\mathbf{s}^{H} \boldsymbol{\Omega} \mathbf{s} / \operatorname{tr}(\boldsymbol{\Omega} \widehat{\mathbf{Z}})$ can be used to measure the approximate accuracy. If the ratio $r$ is close to 1 , it indicates that the approximation is accurate. However, if $r$ is much smaller than 1, we cannot infer that the approximation is inaccurate. The average approximate ratio of the 500 target sets is plotted in the figure versus the number of randomizations $K$. The objective functions of the discrete phase codes are also normalized by the continuous upper bound and shown in the figure for comparison. The desired SNR $d$ equals $0.2 t$ in Figure 4(a) and equals $0.5 t$ in Figure $4(\mathrm{~b})$. We see that the approximate ratios of all the phase codes become larger with increasing $K$ and gradually approach to their limits. With a modest number of randomizations $(K \geq 30)$, the approximate ratio of the continuous phase code reaches 0.997 in the case of $d=0.2 t$ and 0.977 in the case of $d=0.5 t$. Even in the worst case, such approximate accuracy is satisfactory. The discrete phase codes show performance loss when compared to the continuous phase code, since the feasible region is even smaller. As shown in the figure, larger alphabet size leads to higher performance. The objective function increases monotonically with alphabet size $D$. The performance of the phase code with 16 phases is very close to that of the continuous phase code.

\section{Conclusion}

We have proposed and simulated a radar phase-coded waveform design technique for extended target recognition in the presence of colored Gaussian disturbance. A major practical issue, target detection performance, has been considered and solved. The waveform 
was designed to maximize the recognition performance under a control on the achievable SNR of every target hypothesis. Simulation results have highlighted that it is possible to realize a tradeoff between the recognition performance and the detection requirements. With a modest number of randomizations, the objective function achieved by the proposed method was very close to the upper bound obtained from the relaxed problem. Satisfactory approximate accuracy was therefore guaranteed. Moreover, both the performances of continuous and discrete phase codes were compared in the simulation. Statistical results have shown that the discrete phase code with larger phase alphabet size has higher recognition performance, and the performance gradually approaches to the performance of continuous phase code with increasing alphabet size.

\section{Acknowledgments}

This work was supported in part by the National Natural Science Foundation of China (no. 60901057) and the National Basic Research Program of China (973 Program, no. 2010CB731901).

\section{References}

[1] S. Haykin, "Cognitive radar: a way of the future," IEEE Signal Processing Magazine, vol. 23, no. 1, pp. 30-40, 2006.

[2] N. A. Goodman, P. R. Venkata, and M. A. Neifeld, "Adaptive waveform design and sequential hypothesis testing for target recognition with active sensors," Journal on Selected Topics in Signal Processing, vol. 1, no. 1, pp. 105-113, 2007.

[3] A. Wald, "Sequential tests of statistical hypotheses," Annals of Mathematical Statistics, vol. 16, no. 2, pp. 117-186, 1945.

[4] A. Tartakovsky, "Asymptotically optimal sequential tests for nonhomogeneous processes," Sequential Analysis, vol. 17, no. 1, pp. 33-61, 1998.

[5] Mark R. Bell, "Information theory and radar waveform design," IEEE Transactions on Information Theory, vol. 39, no. 5, pp. 1578-1597, 1993.

[6] J. R. Guerci and S. U. Pillai, "Theory and application of optimum transmit-receive radar," in Proceedings of the International radar Conference, pp. 705-710, Washington, DC, USA, 2000.

[7] S. U. Pillai, H. S. Oh, D. C. Youla, and J. R. Guerci, "Optimum transmit-receiver design in the presence of signal-dependent interference and channel noise," IEEE Transactions on Information Theory, vol. 46, no. 2, pp. 577-584, 2000.

[8] J. R. Guerci, Cognitive Radar: The Knowledge-Aided Fully Adaptive Approach, Artech House, 2010.

[9] L. K. Patton and B. D. Rigling, "Modulus constraints in adaptive radar waveform design," in Proceedings of the IEEE International Radar Conference, pp. 1-6, Rome, Italy, 2008.

[10] Y. Wei, H. Meng, and X. Wang, "Adaptive single-tone waveform design for target recognition in cognitive radar," in Proceedings of the IET International Radar Conference, pp. 707-710, Guilin, China, 2009.

[11] J. D. Zhang, X. H. Zhu, and H. Q. Wang, "Adaptive radar phase-coded waveform design," Electronics Letters, vol. 45, no. 20, pp. 1052-1053, 2009.

[12] B. Jiu, H.-W. Liu, L.-Y. Li, and S.-J. Wu, "Waveform design for broadband radar based on phase modulated signal," Journal of Electronics and Information Technology, vol. 30, no. 9, pp. 2038-2041, 2008.

[13] M. I. Skolnik, Radar Handbook Second Edition, McGraw-Hill, New York, NY, USA, 1990.

[14] Yu. Nesterov, "Semidefinite relaxation and nonconvex quadratic optimization," Optimization Methods and Software, vol. 9, no. 1-3, pp. 141-160, 1998.

[15] S. Zhang and Y. Huang, "Complex quadratic optimization and semidefinite programming," SIAM Journal on Optimization, vol. 16, no. 3, pp. 871-890, 2006.

[16] Z.-Q. Luo, W.-K. Ma, A. So, Y. Ye, and S. Zhang, "Semidefinite relaxation of quadratic optimization problems," IEEE Signal Processing Magazine, vol. 27, no. 3, pp. 20-34, 2010.

[17] A. De Maio, S. De Nicola, Y. Huang, S. Zhang, and A. Farina, "Code design to optimize radar detection performance under accuracy and similarity constraints," IEEE Transactions on Signal Processing, vol. 56, no. 11, pp. 5618-5629, 2008. 
[18] A. De Maio, S. De Nicola, Y. Huang, Z.-Q. Luo, and S. Zhang, “Design of phase codes for radar performance optimization with a similarity constraint," IEEE Transactions on Signal Processing, vol. 57, no. 2, pp. 610-621, 2009.

[19] S. Boyd and L. Vandenberghe, Convex Optimization, Cambridge University Press, Cambridge, UK, 2004. 


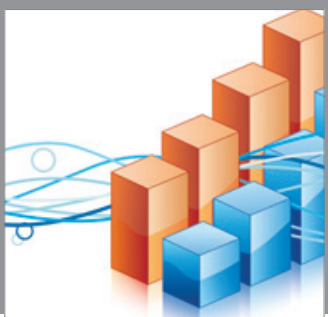

Advances in

Operations Research

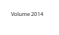

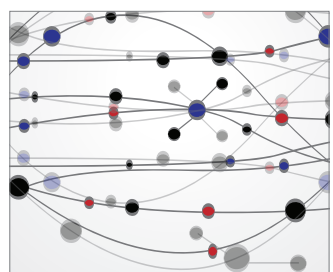

\section{The Scientific} World Journal
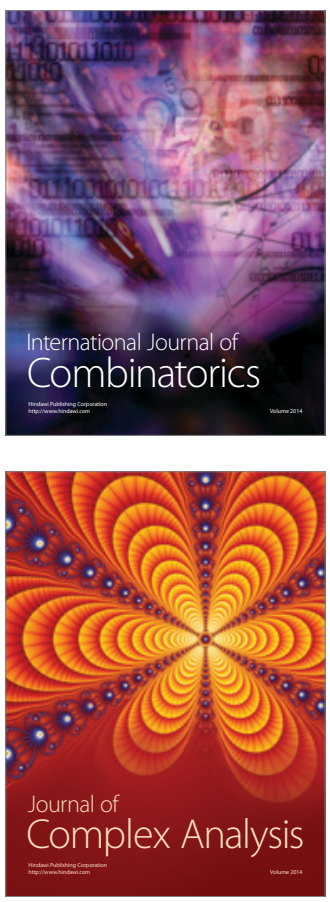

International Journal of

Mathematics and

Mathematical

Sciences
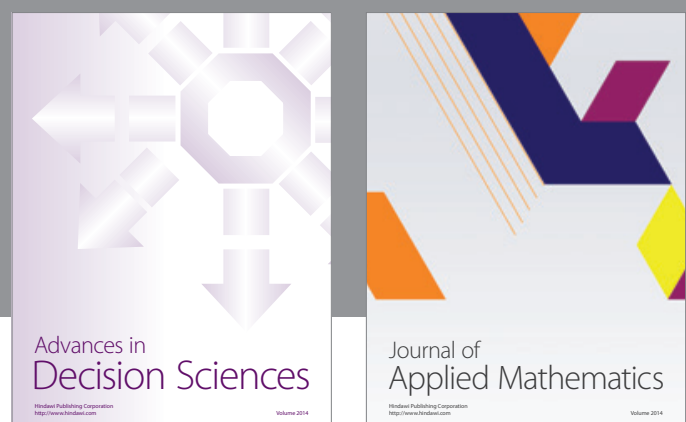

Journal of

Applied Mathematics
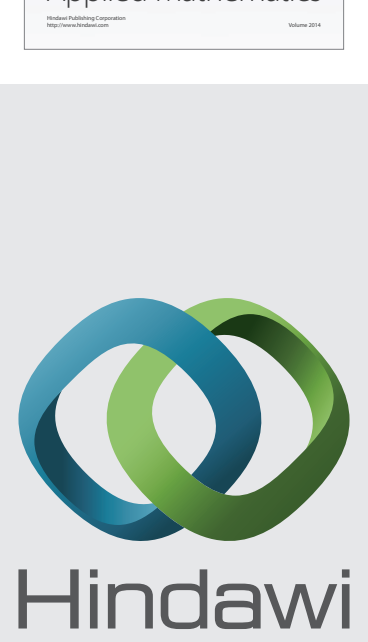

Submit your manuscripts at http://www.hindawi.com
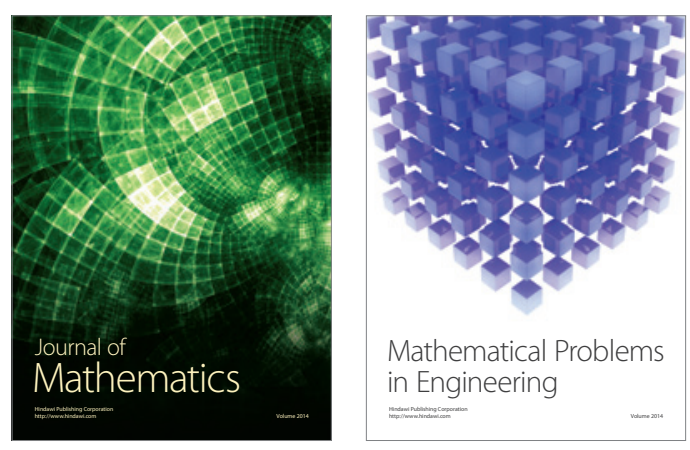

Mathematical Problems in Engineering
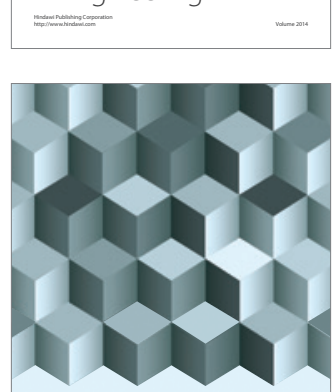

Journal of

Function Spaces
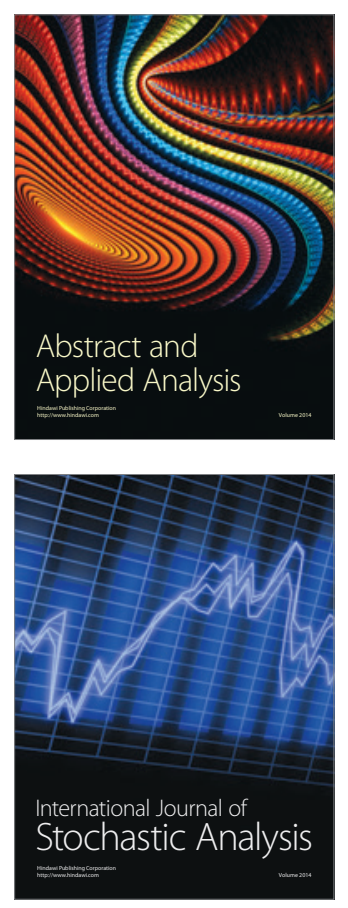

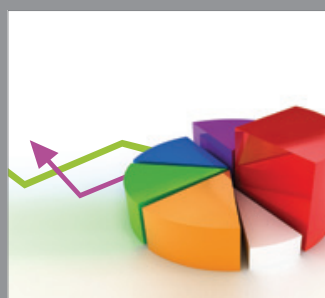

ournal of

Probability and Statistics

Promensencen
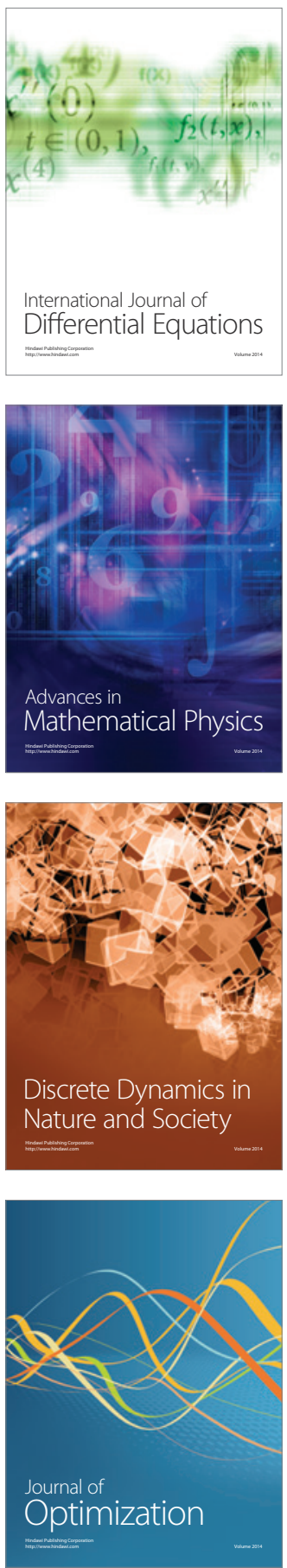